\title{
Critical numbers of attractive Bose-Einstein condensed atoms in asymmetric traps
}

\author{
A. Gammal, ${ }^{1}$ Lauro Tomio, ${ }^{2}$ and T. Frederico ${ }^{3}$ \\ ${ }^{1}$ Instituto de Física, Universidade de São Paulo, 05315-970 São Paulo, Brazil \\ ${ }^{2}$ Instituto de Física Teórica, Universidade Estadual Paulista, 01405-900 São Paulo, Brazil \\ ${ }^{3}$ Departamento de Física, Instituto Tecnológico da Aeronáutica, CTA, 12228-900 São José dos Campos, \\ São Paulo, Brazil
}

(Received 12 June 2002; published 30 October 2002)

\begin{abstract}
The recent Bose-Einstein condensation of ultracold atoms with attractive interactions led us to consider the possibility of probing the stability of its ground state in arbitrary three-dimensional harmonic traps. We performed a quantitative analysis of the critical number of atoms through a full numerical solution of the mean-field Gross-Pitaevskii equation. Characteristic limits are obtained for reductions from three to two and one dimensions, in perfect cylindrical symmetries as well as in deformed ones.
\end{abstract}

DOI: $10.1103 /$ PhysRevA.66.043619

The predicted collapsing behavior of condensed systems with attractive two-body atomic interactions [1], first observed in experiments with ${ }^{7} \mathrm{Li}[2]$, was recently tested in experiments with ${ }^{85} \mathrm{Rb}$ [3]. In the experiments described in Ref. [3], and more recently in [4], by means of Feshbach resonance techniques [5], the two-body interaction was tuned from positive to negative values. In addition to the fact that the experimental results qualitatively agree with the theory, and confirm results of previous variational treatments [6-11], they also show a consistent quantitative deviation of about $20 \%$ from the mean-field predicted critical number of atoms, $N_{c}$ [1]. The asymmetry of the trap was shown in Ref. [12] to be responsible for about $4 \%$ of the observed deviation.

In this respect, it is relevant to obtain precise and reliable numerical results for the mean-field calculations, in order to probe their consistency and possible limitations. The actual experimental atomic traps are in general harmonic and nonsymmetric. Extreme asymmetric traps have been recently employed in experimental investigations with condensates constrained to quasi-one (1D) $[4,13]$ or quasi-two dimensions (2D) [14], exploring the theoretical analysis considered by several authors $[15-17,7,18,19]$. A nonsymmetric threedimensional (3D) trap is reported in Ref. [20], with the frequencies given by $2 \omega_{1}=\sqrt{2} \omega_{2}=\omega_{3}=2 \pi \times 33 \mathrm{~Hz}$.

Considering the general nonsymmetric traps that have been employed, the accuracy of the comparison between experiments and the results of mean-field approximation relies on precise calculations using arbitrary three-dimensional traps. In the case of an attractive two-body interaction, the maximum critical number of atoms for a stable system is one of the interesting observables to study, which is also related to the collapse of the wave function of the system. In these cases, where the two-body scattering length is negative and the kinetic energy cannot be considered to be a small perturbation, the Gross-Pitaevskii mean-field approximation has been applied, giving reliable results in explaining the observations in stable (noncollapsing) conditions [21].

Before presenting the mean-field equation for an arbitrary 3D case, let us analyze qualitatively the collapse phenomenon for asymmetric traps. The interaction energy is proportional to the square of the density, varying with the negative
PACS number(s): 03.75.Fi, 32.80.Pj, 42.65.Tg, 02.60.Jh

two-body scattering length. For traps with cylindrical (or almost cylindrical) shapes, there are two quite different situations: one pancakelike, with the frequencies in the transverse directions being smaller than the frequency in the longitudinal direction; the other, cigarlike (quasi-1D), with the frequency in the longitudinal direction smaller than the frequencies in the perpendicular directions. For a true 1D system, one does not expect the collapse of the system with increasing number of atoms $[1,22]$. However, it happens that a realistic 1D limit is not a true 1D system, with the density of particles still increasing due to the strong restoring forces in the perpendicular directions [16]. The relevance of quasi-1D traps in controlling the condensate motion has been pointed out in Ref. [16]. But, as we are going to see, the critical number of particles in the quasi-1D limit is smaller than the critical number of particles in the 2D limit, if we just exchange the longitudinal and perpendicular frequencies. The physical reason for that behavior is the increase of the average density in the cigarlike configuration relative to the pancake like one for the same number of atoms, implying a strong collapsing force in the first case, and consequently the cigarlike geometry is a more unstable configuration compared to the pancake like one. This conclusion is in apparent contradiction with the remark made in Sec. IV of Ref. [16], saying that, considering the better collapse-avoiding properties, "the cigar-shaped trap is the optimal one." We are going to discuss this problem in detail and clarify this issue.

In the following, we revise the Gross-Pitaevskii (GP) formalism, for an atomic system with arbitrary nonspherically symmetric harmonic trap. The Bose-Einstein condensate, at zero temperature, in the GP mean-field approximation is given by

$$
\begin{aligned}
\mathrm{i} \hbar \frac{\partial}{\partial t} \Psi(\vec{r}, t)= & {\left[-\frac{\hbar^{2}}{2 m} \nabla^{2}+\frac{m}{2}\left(\omega_{1}^{2} r_{1}^{2}+\omega_{2}^{2} r_{2}^{2}+\omega_{3}^{2} r_{3}^{2}\right)\right.} \\
& \left.+\frac{4 \pi \hbar^{2} a}{m}|\Psi(\vec{r}, t)|^{2}\right] \Psi(\vec{r}, t)=\mu \Psi(\vec{r}, t),
\end{aligned}
$$

where $r=\sqrt{r_{1}^{2}+r_{2}^{2}+r_{3}^{2}}, \quad m$ is the mass of the atom, $\mu$ is the chemical potential, and the wave function $\Psi \equiv \Psi(\vec{r}, t)$ $=\Psi(\vec{r}, 0) \exp (-\mathrm{i} \mu t / \hbar)$ is normalized to the number of par- 
ticles $N$. The arbitrary geometry of the trap is parametrized by three different frequencies $\omega_{1}, \omega_{2}$, and $\omega_{3}$. For convenience, it is appropriate to define the frequencies according to their magnitude, such that in the present work we assume $\omega_{1} \leqslant \omega_{2} \leqslant \omega_{3}$.

Here we will be concerned only with systems that have attractive two-body interactions $[a=-|a|$, in Eq. (1)]. In this case, it was first shown numerically in Ref. [1] that the system becomes unstable if a maximum critical number of atoms, $N_{c}$, is achieved. We present precise results for a critical parameter $k$, directly related to the maximum number of atoms, in a general nonsymmetric configuration of the trap.

By rewriting Eq. (1) in dimensionless units,

$$
i \frac{\partial \phi}{\partial \tau}=\left[\frac{1}{2} \sum_{i}\left(-\frac{\partial^{2}}{\partial x_{i}^{2}}+\frac{\omega_{i}^{2} x_{i}^{2}}{\omega^{2}}\right)-|\phi|^{2}\right] \phi,
$$

where $\tau \equiv \omega t, r_{i} \equiv l_{0} x_{i}$, and $\phi \equiv l_{0} \sqrt{4 \pi|a|} \Psi$, with

$$
\int d^{3} x|\phi|^{2}=4 \pi \frac{N|a|}{l_{0}}
$$

The oscillator length $l_{0}$ is defined in terms of $\omega$, which is taken as the geometrical mean value of the frequencies:

$$
l_{0} \equiv \sqrt{\frac{\hbar}{m \omega}}, \text { with } \omega \equiv\left(\omega_{1} \omega_{2} \omega_{3}\right)^{1 / 3} .
$$

For strongly nonsymmetric cases, particularly when comparing the two extreme cylindrical-shape geometries $\omega_{1} \sim \omega_{2}$ $\ll \omega_{3}$ (pancake shape) and $\omega_{1} \ll \omega_{2} \sim \omega_{3}$ (cigar shape), a noticeable difference is expected between the corresponding critical numbers of particles.

We define a parameter $k$, related to the critical number of trapped atoms, $N_{c}$, as in Ref. [3]:

$$
k=\frac{N_{c}|a|}{l_{0}} .
$$

This parameter is a maximum critical limit for stable solutions of the dimensionless Eq. (2). It depends only on the ratio of the frequencies of the trap. Within the precision given in Ref. [23], $k_{s}=0.5746$, where $k_{s}$ is $k$ for spherically symmetric traps. In Ref. [24], the critical number was calculated for a nonsymmetrical geometry, but in a case where the frequency ratio is not too far from unity $\left(\omega_{1} / \omega_{\perp}=0.72\right)$, giving a result for the number of atoms almost equal to the spherical one.

In experiments with ${ }^{85} \mathrm{Rb}$ [3], an almost cylindrical "cigar-type" symmetry was considered, with the three frequencies given by $17.47 \mathrm{~Hz}, 17.24 \mathrm{~Hz}$ and $6.80 \mathrm{~Hz}$. With this symmetry, the authors have obtained $k=0.459 \pm 0.012$ (statistical) \pm 0.054 (systematic). In Ref. [12], assuming the cylindrical symmetry $\omega_{1}=2 \pi \times 6.80 \mathrm{~Hz}, \omega_{2}=\omega_{3}=2 \pi$ $\times 17.35 \mathrm{~Hz}, k=0.55$ was obtained, a value about $4 \%$ lower than $k_{s}$.

In our numerical approach, the calculation is performed by evolving the nonlinear equation (2) through imaginary time [24]. The evolution is performed for an initial value of

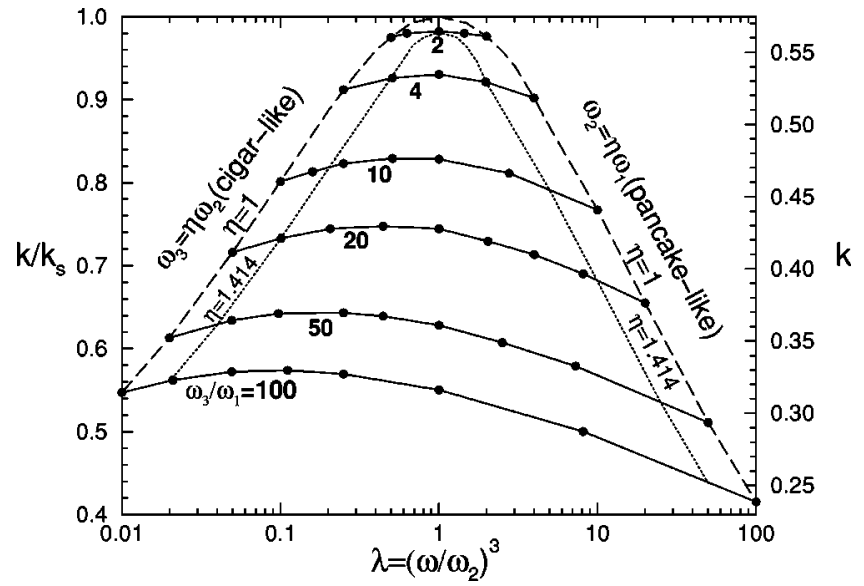

FIG. 1. Critical constant $k=N_{c}|a|(m \omega / \hbar)^{1 / 2}$, calculated for an arbitrary nonsymmetric trap, $\omega \equiv\left(\omega_{3} \omega_{2} \omega_{1}\right)^{1 / 3}$, with $\omega_{3} \geqslant \omega_{2} \geqslant \omega_{1}$. The ratio $\omega_{3} / \omega_{1}$ is shown below each corresponding curve (solid lines, with full circles). The dashed and dotted lines correspond to cylindrical $(\eta=1)$ and deformed cylindrical $(\eta=\sqrt{2})$ symmetries, respectively.

the normalization (3) until the wave function relaxes to the ground state. The wave function is renormalized after each time step. The process is repeated systematically for larger values of the normalization, until a critical limit is reached. At this critical limit the ground state becomes unstable. The time evolution is done with a semi-implicit second order finite difference algorithm. An alternating scheme is used in the $x_{1}$ and $x_{2}$ directions, with a split step in the $x_{3}$ direction. This procedure is done only for $x_{i} \geqslant 0$, taking advantage of the reflection symmetry of the ground state. We consider $100^{3}$ grid points and a time step equal to $\Delta \tau=0.001$, verifying that the algorithm is stable for long time evolution. As we increment the normalization, approaching the critical limit, the wave function starts to shrink. So, in order to maintain the precision, we introduce an automatic reduction of the grid sizes $\Delta x_{1}, \Delta x_{2}$, and $\Delta x_{3}$, gauged by the respective root-mean-square radius in each direction.

In Fig. 1 we show our main results for the critical constant $k$, covering many different geometries. We plot $k / k_{s}$ (and $k$ on the right-hand $y$ axis) as a function of $\lambda$, which is defined by

$$
\lambda \equiv \frac{\omega_{1} \omega_{3}}{\omega_{2}^{2}}=\left(\frac{\omega}{\omega_{2}}\right)^{3} \text { with } \omega_{3} \geqslant \omega_{2} \geqslant \omega_{1} .
$$

We show several curves in which we kept constant the ratio $\omega_{3} / \omega_{1}$ (solid lines). The values of $\omega_{3} / \omega_{1}$ are indicated inside the figure, just below the corresponding plot. The dashed and dotted lines correspond to cylindrical $(\eta=1)$ and deformed cylindrical $(\eta=1.414)$ symmetries for the trap, where $\eta$ is the deformation parameter, as will be explained. In the left-hand side of these plots we have $\omega_{3}=\eta \omega_{2} \gg \omega_{1}$ (cigar shape); and, in the right-hand side $\omega_{3} \gg \omega_{2}=\eta \omega_{1}$ (pancake shape). $k$ can be determined for any symmetry that was not shown, by interpolating the results already given. The results for the complete nonsymmetric case are consistent with the previously obtained values in cylindrical sym- 


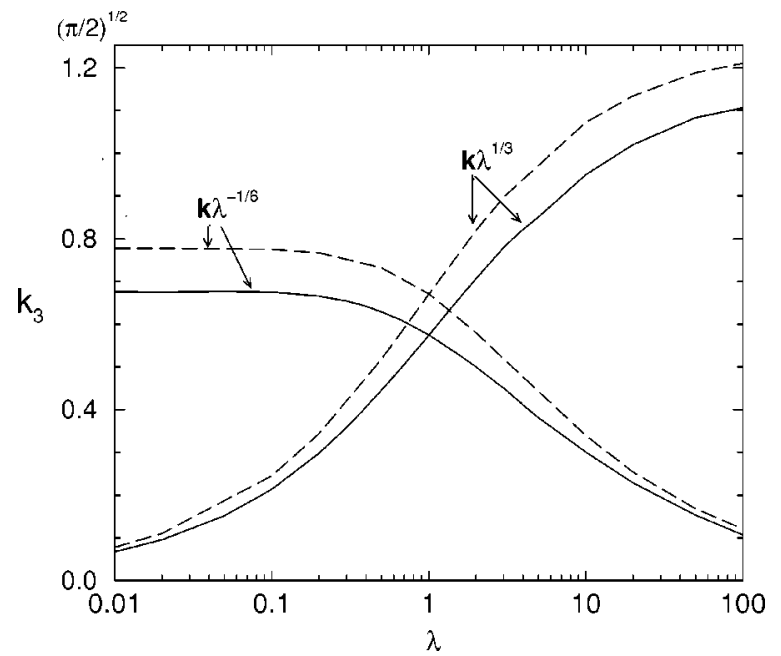

FIG. 2. Exact results of $k_{3} \equiv N_{c}|a|\left(m \omega_{3} / \hbar\right)^{1 / 2}$ for cylindrical traps (solid lines) are compared with corresponding variational approaches (dashed lines). In the 2D pancake limit, $\omega_{\perp}=\omega_{2}=\omega_{1}$ and $k_{3}=k \lambda^{1 / 3}$; and, in the quasi-1D cigar limit, $\omega_{\perp}=\omega_{2}=\omega_{3}$ and $k_{3}$ $=k \lambda^{-1 / 6}$. Note that $\lambda=\omega_{3} / \omega_{1}$ when $\omega_{2}=\omega_{1}$; and $\omega_{1} / \omega_{3}$ when $\omega_{2}=\omega_{3}$.

metry [12]. The maximum value for the critical number $k$ is obtained for the spherically symmetric case $\left(\omega_{1}=\omega_{2}=\omega_{3}\right)$.

As already observed, Fig. 1 includes previous calculations in the limit of the quasi-1D (cigar-shape) and quasi-2D (pancake-shape) symmetries (see dashed line). However, for the sake of comparison with previous results obtained by several authors, we also present in Fig. 2 the cylindrical pancake-type $\left(\omega_{\perp}=\omega_{1}=\omega_{2} \ll \omega_{3}\right)$ and cigar-type $\left(\omega_{\perp}=\omega_{3}\right.$ $\left.=\omega_{2} \gg \omega_{1}\right)$ results. In Fig. 2, for cylindrical geometries, we compare our exact results for $k_{3}=N_{c}|a|\left(m \omega_{3} / \hbar\right)^{1 / 2}$ as a function of $\lambda$ with the corresponding variational ones of Refs. $[7,15]$. The variational results (dashed lines) are consistently a bit higher than the exact ones. For $\lambda \rightarrow 0\left(\omega_{1}\right.$ $\left.\ll \omega_{2}=\omega_{3}=\omega_{\perp}\right)$, the exact and variational results for the critical constant are $N_{c}|a|\left(m \omega_{\perp} / \hbar\right)^{1 / 2}=0.676$ and 0.776 , respectively. They are consistent with the quasi-1D limits given in Refs. $[15,16]$. When $\omega_{3} \gg \omega_{2}=\omega_{1}$, the variational 2D limit $\sqrt{\pi / 2}$ of Ref. [7] is comparable with our exact result $\left(k_{3}=0.931 \sqrt{\pi / 2}\right)$. In this case, the quasi-2D limit coincides with the true $2 D$ limit $[25,19,22,26]$.

Considering the present analysis, we observe that $N_{c}$ in a cigar-like (quasi-1D) trap is smaller than $N_{c}$ in a pancakelike (quasi-2D) trap. And, as we deform a cigarlike trap, $N_{c}$ also increases. We need to clarify this matter, which is the subject of the next four paragraphs, because this result apparently contradicts a remark made in Ref. [16], saying that "the cigar-shaped trap is the optimal one."

As shown in Ref. [12] and also in the present calculation, in a deformed cylindrical symmetry, the cigar shape (with one of the frequencies smaller than the other two) is more favorable to obtain a larger value of $k$ than the pancake-shape symmetry (with one of the frequencies larger than the other two). See, for example, in Fig. 1, the two extreme points of the curve with $\omega_{3} / \omega_{1}=100$. This results from the definition of $k$, Eq. (5), in terms of the average oscillator length $l_{0}$.
However, the maximum value of $k$ can only be directly related to the maximum value of $N_{c}$ when $\omega$ is kept fixed. And, with $l_{0}$ fixed, $N_{c}$ is maximized for $\lambda=1$, corresponding to the spherically symmetric case $\left(k=k_{s}\right)$. If we fix $l_{0}$ and $\omega_{3} / \omega_{1}, N_{c}$ is maximized for a deformed cylindrical symmetry with $\lambda<1$, as one can see from Fig. 1 .

Considering exact cylindrical traps, by exchanging the frequencies (which, obviously, does not keep constant the average frequency $\omega$ ), the following ratio that was obtained in Ref. [12] is valid:

$$
R(\lambda) \equiv \frac{N_{c}(\lambda)}{N_{c}(1 / \lambda)}=\lambda^{1 / 6} \frac{k(\lambda)}{k(1 / \lambda)} .
$$

This result favors the pancakelike symmetry $\left(\lambda=\omega_{3} / \omega_{1}\right.$ $=\omega_{3} / \omega_{\perp}>1$ ), to obtain a larger value for $N_{c}$. Consider, for example, a cylindrical pancake-type trap with $\lambda=100$, in comparison with a cigar-type trap with $\lambda=1 / 100$. We notice that, in this case, $R(\lambda=100) \approx 1.6$, implying that with such pancakelike trap $\left(\omega_{3}=100 \omega_{\perp}\right)$ one can obtain about $60 \%$ more particles than with the corresponding cigarlike trap $\left(\omega_{3}=\omega_{\perp}=100 \omega_{1}\right)$. Let us consider the recent experiment with a quasi-1D (cigarlike) trap used in the formation and propagation of matter wave solitons, with ${ }^{7} \mathrm{Li}$ [4]. In this case, axial and radial frequencies, respectively, equal to 3.2 $\mathrm{Hz}$ and $\sim 625 \mathrm{~Hz}$ were used [29]; or $\omega_{\perp}=\omega_{3}=2 \pi$ $\times 625 \mathrm{~Hz}, \omega_{1}=2 \pi \times 3.2 \mathrm{~Hz}$, and $\lambda=\omega_{1} / \omega_{3}=0.00513$. So, as shown in Fig. 2, we are essentially in the limit $\lambda=0$, which gives $N_{c}^{1} \mathrm{D}|a| / \sqrt{\hbar /\left(m \omega_{\perp}\right)} \approx 0.675$. Considering that the scattering length was tuned to $a=-3 a_{0}$ ( $a_{0}$ is the Bohr radius), the maximum number of atoms in this quasi-1D trap is $N_{c}^{1 D} \approx 6400$. If we exchange the radial and axial frequencies in this experiment, going from a cigarlike to a pancakelike trap, $\omega_{\perp}=2 \pi \times 3.2 \mathrm{~Hz}, \omega_{3}=2 \pi \times 625 \mathrm{~Hz}$, and $\lambda$ $=\omega_{3} / \omega_{\perp}=195.3$. In this case, $N_{c}^{2} \mathrm{D}|a| / \sqrt{\hbar /\left(m \omega_{3}\right)} \approx 1.12$. So the critical number in the quasi-2D limit is about $66 \%$ larger than the corresponding number of atoms in the quasi-1D limit, and $N_{c}^{2} \mathrm{D} \approx 10600$.

The best way to distribute the frequencies to obtain the maximum number of atoms was first considered in Ref. [6], reaching the conclusion that the best is a spherical trap for achieving maximum density. (In an almost spherical trap authors obtained from $\sim 600$ to $\sim 1300$ atoms, in overall agreement with theoretical predictions [2].) The point is that to say what is the best configuration for the maximum number of atoms we must also say what is the constraint on the frequencies. If the constraint is to keep fixed the product $\omega_{1} \omega_{2} \omega_{3}$ then the maximum number will be given for the maximum $k$ [as shown in Eq. (5)], which happens in spherical symmetry, in agreement with Ref. [6]. But, if two frequencies are equal and given a priori, then the best configuration to increase $N_{c}$ is to make the third frequency equal to zero, in agreement with Ref. [16]. However, if only one frequency is kept fixed, than the extreme pancake shape will contain more atoms, by making the remaining frequencies go to zero.

As a final remark about the maximization of $N_{c}$, we note that, for attractive two-body interactions, the frequencies in 
all three directions cannot be initially zero. In the experimental process of condensation, the atoms must be trapped while going from higher temperature $T$ to the critical one $T_{c}$. By making the frequencies too small, $T_{c}$ will be smaller and the condensation harder to achieve [as shown in Ref. [27], $T_{c}$ $\sim \hbar \omega\left(N_{t o t}\right)^{1 / 3}$, where $N_{t o t}$ is the total number of particles in the trap]. Once the condensate is achieved, we can switch off some of the frequencies, and obtain a finite maximum value for $N_{c}$, as observed in Fig. 2 for $T=0$. In the present work, we are concerned with the case of zero temperature. The effects of temperature on the collapse and on $N_{c}$ for the spherically symmetric case have been discussed, for example, in Ref. [28]. $N_{c}$ is maximized by making $\omega$ as small as possible. However, a discontinuity exists when taking all the three frequencies exactly zero. Without a trap a collapse will occur for any number of particles.

We must stress that Fig. 1 also includes nonsymmetric cigar-type geometries $\omega_{3}=\eta \omega_{2} \gg \omega_{1}$ and elliptical pancaketype geometries $\omega_{3} \gg \omega_{2}=\eta \omega_{1}$, where $\eta$ is the eccentricity of the ellipses. In particular, the dotted line in Fig. 1 gives the critical values of $k$ for $\eta=\sqrt{2}$. Thus, one could replot, as in Fig. 2, a series of curves whose limiting cases describe nonsymmetric cigar- or pancakelike symmetries. In the 1D and 2D limits, one can obtain critical quasi-1D and quasi-2D numbers, for each $\eta$.

One can also obtain an interesting result for asymmetric cigar-shape traps, where $\omega_{3}=\eta \omega_{2} \gg \omega_{1}$, following the approach given in Sec. II of Ref. [16]. The assumption made in [16] to reduce the $3 \mathrm{D}$ equation to a $1 \mathrm{D}$ equation can be justified in the limit when the forces in the transverse directions are the main forces responsible for the trapping potential. So, in the limit $\omega_{1} \rightarrow 0$, we are able to generalize the solitonic 1D equation for a cigar-shaped trap, deformed by a given ratio $\eta=\omega_{3} / \omega_{2}$ between the transverse direction frequencies. In this case, $\eta \geqslant 1$ cannot be arbitrarily larger. Also, by a scaling procedure applied to the equation for different values of $\eta$, using our previous exact result, we have

$$
N_{c}|a|\left(\frac{m \sqrt{\omega_{2} \omega_{3}}}{\hbar}\right)^{1 / 2}=\frac{N_{c}|a|}{\eta^{1 / 4}} \sqrt{\frac{m \omega_{3}}{\hbar}}=0.676 .
$$

This generalizes the cigar-shape quasi-1D results of [16] (with $\eta$ such that we still have $\omega_{2} \gg \omega_{1}$ ). The 1D solid line given in Fig. 2 is also applied to deformed cigar-shaped symmetries if we replace $k_{3}$ by $k_{3} \eta^{-1 / 4}$ on the $y$ axis. This result may be relevant for asymmetric waveguide propagation as one can deform the cigar-type symmetry and control the collapsing condition. From Eq. (8) we observe that the maximum critical number $N_{c}$ will increase when the cigarlike symmetry is deformed by a factor proportional to $\left(\omega_{3} / \omega_{2}\right)^{1 / 4}$.

Now, let us see the effect of deformation in the same example of the cigar-shaped geometry used in Ref. [4], with ${ }^{7} \mathrm{Li}$ gas. We use the same value of $a=-3 a_{0}$, with $\omega_{3}$ $=2 \pi \times 625 \mathrm{~s}^{-1}$, and take $\omega_{2}=\omega_{3} / \eta$. As given in Eq. (8), we go from $N_{c}=6392(\eta=1)$ to $N_{c}=6970(\eta=\sqrt{2})$ or $N_{c}$ $=7601(\eta=2)$. This means an increase of $\sim 9 \%$ when $\eta$ $=\sqrt{2}$ and $\sim 19 \%$ when $\eta=2$. When $\eta \gg 1$, the approxima-

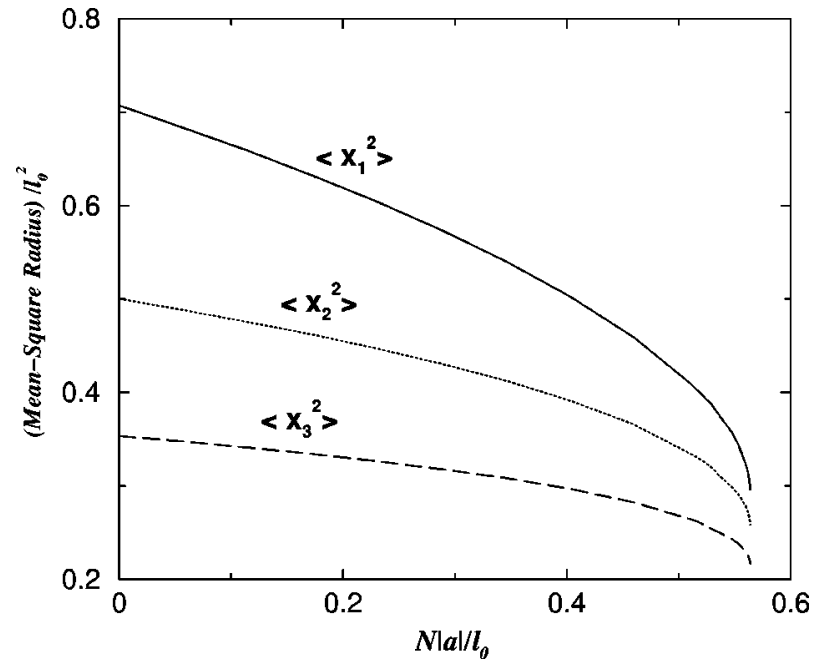

FIG. 3. The three components $i=1,2,3$ of the mean-square radius $\left\langle x_{i}^{2}\right\rangle=\left\langle r_{i}^{2}\right\rangle / l_{0}^{2}$ are shown in terms of the dimensionless $N|a| / l_{0}$, for the case that $\omega_{3}=\sqrt{2} \omega, \omega_{2}=\omega$, and $\omega_{1}=\omega / \sqrt{2}$.

tion considered in the wave-function separation, as given in [16], is not valid. In this case we reach the other deformed pancakelike symmetry, where $\omega_{3} \gg \omega_{2} \sim \omega_{1}$. However, in the pancake geometry, the effect of deformation in $N_{c}$ is negligible. Comparing the quasipancakelike geometry with the quasicigarlike geometry shows that the number $N_{c}$ in the cigarlike geometry is much more sensitive to deformations.

We have also studied the behavior of the root-meansquare radius for the case $\omega_{3}=\sqrt{2} \omega_{2}=2 \omega_{1}$. We verified that, as the system approaches the critical point (or collapse), the wave function tends to be more "spherical," confirming an earlier conclusion made with a Gaussian variational approximation [30]. In Fig. 3, we show the corresponding results, for the three components of the mean-square radius $\left\langle r_{i}^{2}\right\rangle=l_{0}^{2}\left\langle x_{i}^{2}\right\rangle(i=1,2,3)$. As shown, when $N=0$, we have $\left\langle r_{1}^{2}\right\rangle /\left\langle r_{2}^{2}\right\rangle=\left\langle r_{2}^{2}\right\rangle /\left\langle r_{3}^{2}\right\rangle=\sqrt{2}$; and when $N \approx N_{c}$ this ratio is drastically reduced.

In summary, we have calculated systematically the critical number of particles, in systems that have negative two-body interactions, for traps with arbitrary geometries. The maximum critical number of particles, $N_{c}$, can be derived from the given value of the parameter $k$, given in Eq. (5), once one has the scattering length, the mass of the atomic system, and the frequencies of the trap. The results are shown in Figs. 1 and 2. The value of $k$, for any symmetry not explicitly given, can be easily derived from Fig. 1 by interpolation. It is also pointed out that the results shown in Fig. 2, in the 1D cigarlike case, can be extended for slightly deformed cylindrical symmetries, by replacing the $y$ axis label $k_{3}$ by $k_{3} \eta^{-1 / 4}$.

Our main results in the present work are as follows. (i) The maximum number of particles $N_{c}$ for arbitrary 3D trap geometries is given through the results shown in Fig. 1. (ii) The optimal trap configuration, to avoid collapse with maximum $N_{c}$, is found to be strongly dependent on the constraints of the frequencies of the trap. If we initially fix one of the frequencies, the best configuration of the trap is pancakelike, with the other two frequencies going to zero. Analogously, if we initially fix two equal frequencies, the 
best configuration of the trap is cigarlike, with the third frequency close to zero. If we initially fix two different frequencies and try to vary the third frequency between the fixed ones, the best configuration is again pancakelike. We show that $N_{c}$ is much more sensitive to deformations of the trap in a cigarlike geometry than in a pancakelike geometry. Finally, for small deformations $\eta$ of the cigarlike traps, where $\eta$ $\geqslant 1$, releasing the longitudinal direction, the solitonic solutions obtained in Ref. [16] will be rescaled by the deforma- tion. $N_{c}$ will be rescaled by a factor of $\eta^{1 / 4}$, generalizing the findings of Ref. [16].

We are grateful to Randy Hulet for information provided. A.G. also thanks Emerson Passos and Marcelo Pires for useful discussions. For partial support, we thank Fundação de Amparo à Pesquisa do Estado de São Paulo (FAPESP) and Conselho Nacional de Desenvolvimento Científico e Tecnológico $(\mathrm{CNPq})$.
[1] P. A. Ruprecht, M. J. Holland, K. Burnett, and M. Edwards, Phys. Rev. A 51, 4704 (1995).

[2] C. C. Bradley, C. A. Sackett, J. J. Tollett, and R. G. Hulet, Phys. Rev. Lett. 75, 1687 (1995); C. C. Bradley, C. A. Sackett, and R. G. Hulet, ibid. 78, 985 (1997); J. M. Gerton, D. Strekalov, I. Prodan, and R. G. Hulet, Nature (London) 408, 692 (2000).

[3] J. L. Roberts et al., Phys. Rev. Lett. 86, 4211 (2001); E. Donley et al., Nature (London) 412, 295 (2001).

[4] K. E. Strecker, G. B. Partridge, A. G. Truscott, and R. G. Hulet, Nature (London) 417, 150 (2002).

[5] S. Inouye et al., Nature (London) 392, 151 (1998); Ph. Courteille, R. S. Freeland, D. J. Heinzen, F. A. van Abeelen, and B. J. Verhaar, Phys. Rev. Lett. 81, 69 (1998).

[6] C. A. Sackett, C. C. Bradley, M. Welling, and R. G. Hulet, Appl. Phys. B: Lasers Opt. 65, 433 (1997).

[7] G. Baym and C. J. Pethick, Phys. Rev. Lett. 76, 6 (1996).

[8] E. V. Shuryak, Phys. Rev. A 54, 3151 (1996).

[9] H. T. C. Stoof, J. Stat. Phys. 87, 1353 (1997).

[10] M. Ueda and A. J. Leggett, Phys. Rev. Lett. 80, 1576 (1998).

[11] M. Wadati and T. Tsurumi, J. Phys. Soc. Jpn. 68, 3840 (1999).

[12] A. Gammal, T. Frederico, and L. Tomio, Phys. Rev. A 64, 055602 (2001).

[13] F. Schreck et al., Phys. Rev. Lett. 87, 080403 (2001); Phys. Rev. A 64, 011402 (2001).

[14] A. Görlitz et al., Phys. Rev. Lett. 87, 130402 (2001).

[15] V. M. Pérez-Garcia, H. Michinel, J. I. Cirac, M. Lewenstein, and P. Zoller, Phys. Rev. A 56, 1424 (1997).

[16] V. M. Pérez-Garcia, H. Michinel, and H. Herrero, Phys. Rev. A 57, 3837 (1998).

[17] L. D. Carr, C. W. Clark, and W. P. Reinhardt, Phys. Rev. A 62, 063610 (2000); 62, 063611 (2000); L. D. Carr, M. A. Leung, and W. P. Reinhardt, J. Phys. B 33, 3983 (2000).

[18] R. K. Bhaduri, S. M. Reimann, S. Viefers, A. G. Choudhury, and M. K. Srivastava, J. Phys. B 33, 3895 (2000).

[19] S. K. Adhikari, Phys. Rev. E 62, 2937 (2000).

[20] W. K. Hensinger et al., Nature (London) 412, 52 (2001).

[21] J. R. Anglin and W. Ketterle, Nature (London) 416, 211 (2002).

[22] A. Gammal, T. Frederico, L. Tomio, and F. K. Abdullaev, Phys. Lett. A 267, 305 (2000).

[23] A. Gammal, T. Frederico, and L. Tomio, Phys. Rev. E 60, 2421 (1999).

[24] F. Dalfovo and S. Stringari, Phys. Rev. A 53, 2477 (1996).

[25] M. I. Weinstein, Commun. Math. Phys. 87, 567 (1983).

[26] L. Bergé, T. J. Alexander, and Y. S. Kivshar, Phys. Rev. A 62, 023607 (2000).

[27] F. Dalfovo, S. Giorgini, L. P. Pitaevskii, and S. Stringari, Rev. Mod. Phys. 71, 463 (1999).

[28] M. J. Davis, D. A. W. Hutchinson, and E. Zaremba, J. Phys. B 32, 3993 (1999).

[29] R. G. Hulet (private communication).

[30] M. O. D. Pires and E. J. V. de Passos, J. Phys. B 33, 3929 (2000). 